\title{
Spatial Separation of Plasmonic Hot Electron Generation and a Hydrodehalogenation Reaction Center Using a DNA Wire
}

Sergio Kogikoski Jr. ${ }^{\text {a, }}$, Anushree Dutta ${ }^{\text {a }}$, Ilko Bald ${ }^{\mathrm{a}^{*}}$

a Institute of Chemistry, Physical Chemistry, University of Potsdam, Karl-Liebknecht-Str. 2425, 14476, Potsdam, Germany.

${ }^{b}$ Department of Analytical Chemistry, Institute of Chemistry, State University of Campinas (UNICAMP), P.O. Box 6154, 13083-970, Campinas-SP (Brazil).

*e-mail: kogikoskijunior@uni-potsdam.de bald@uni-potsdam.de

Tel: +49 331-977-6114 (SKJr)

+49 331-977-5238 (IB) 


\begin{abstract}
Using hot charge carriers far from a plasmonic nanoparticle surface is very attractive for many applications in catalysis and nanomedicine, and will lead to a better understanding of plasmon-induced processes, such as hot charge carrier or heat driven chemical reactions. Herein we show that DNA is able to transfer hot electrons generated by a silver nanoparticle over several nanometers to drive a chemical reaction in a molecule non-adsorbed on the surface. For this we use 8-bromo-adenosine introduced in different positions within a double stranded DNA oligonucleotide. The DNA is also used to assemble the nanoparticles into superlattices enabling the use of surface enhanced Raman scattering to track the decomposition reaction. To prove the DNA mediated transfer, the probe molecule was insulated from the charge carriers source, which hindered the reaction. The results indicate that DNA can provide an attractive platform to study the transfer of hot electrons, leading to the future development of more advanced plasmonic catalysts.
\end{abstract}

Keywords: Plasmonics, DNA nanotechnology, Hot-electrons, Charge transfer, SERS, Superlattices 


\section{TOC}

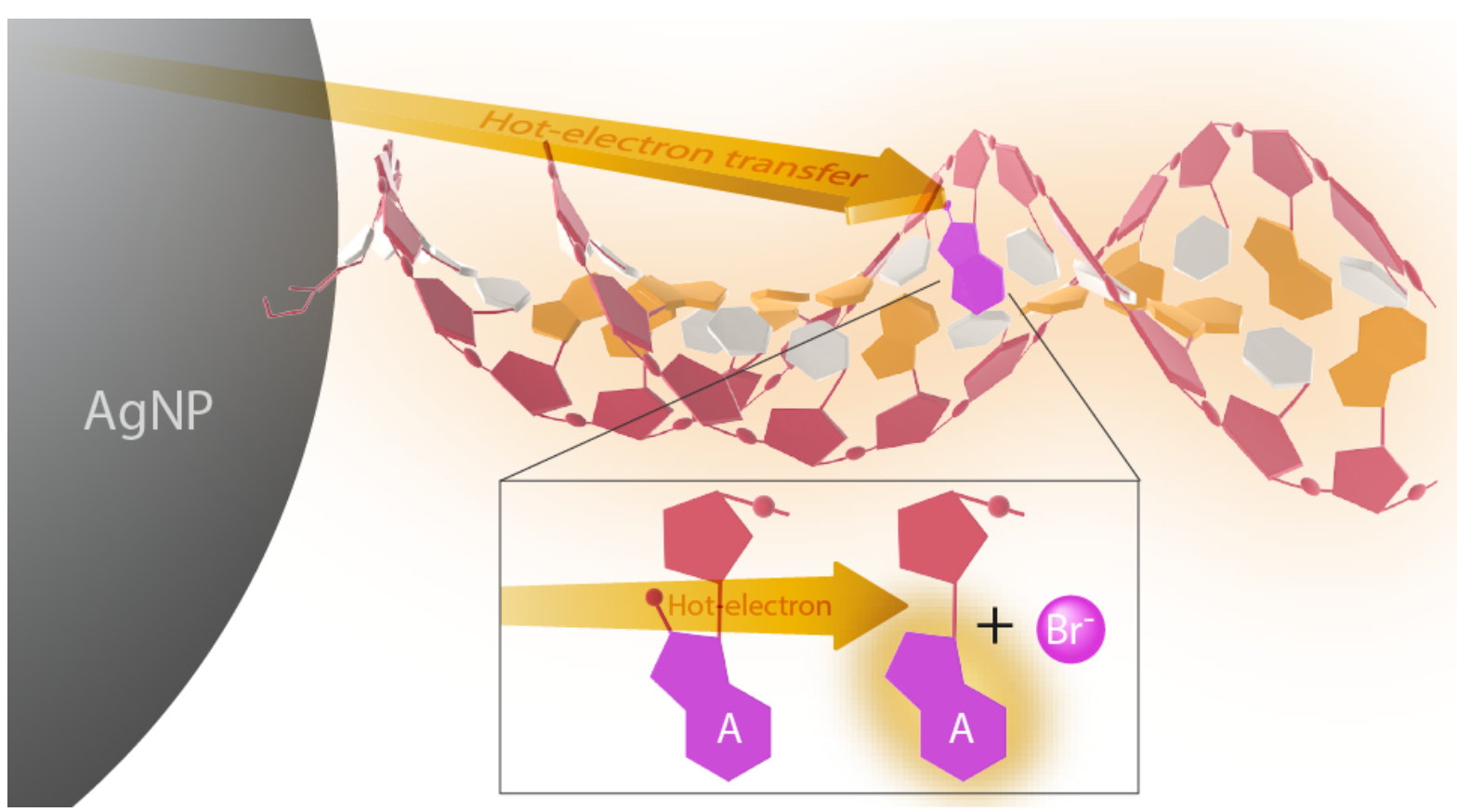




\section{Introduction}

One of the most interesting and promising uses of plasmonic nanoparticles is the possibility to induce chemical reactions at their interface giving rise to the emerging field of plasmon chemistry. The reactions are driven by different processes occurring at the interface between the plasmonic nanoparticle and the molecules, such as the generation of hot-carriers and also the thermalization of these carriers into heat. Even though it is very difficult to distinguish the contribution of the two mechanisms, both are suggested to affect the reaction pathways to some extent. ${ }^{1,2}$ Anyway, for both cases there is the necessity of close contact of the reagent molecule with the nanoparticle surface, i.e., covalent bonding or adsorption, to activate it to further undergo the reaction. ${ }^{3,4}$ This interaction can be problematic leading principally to the inactivation of the nanoparticle surface due to surface poisoning or undesired product generation, such as amorphous carbon..$^{5-8}$

So far, many molecules were already shown to react on the surface of illuminated plasmonic nanoparticles, like $\mathrm{CO}_{2}, \mathrm{H}_{2}$, 4-nitrothiophenol and other organic molecules which were recently reviewed by Gellé et al. ${ }^{9}$ The case of the dimerization of 4-nitrothiophenol is the most studied since the reaction can be directly tracked using surface enhanced Raman spectroscopy (SERS), which can also be used to study the reaction mechanism. Nevertheless, the mechanisms that trigger and direct the reaction pathways either by hot-carriers or by heat are still under debate since the reaction involves many different steps, and at least 8 electrons to proceed. ${ }^{2,10,11}$ Our group recently has shown that brominated nucleobases can undergo a plasmon induced reduction when adsorbed onto gold or silver nanoparticles, and that the reaction can be tracked using SERS. ${ }^{12,13}$ The hot-electrons generated on the nanoparticles are transferred to the brominated nucleobase which is followed by cleavage of the $\mathrm{C}-\mathrm{Br}$ bond generating the non-brominated base, in a procedure that only requires one electron and one proton via a dissociative electron attachment (DEA) mechanism. ${ }^{14}$ Here we study this reaction with the brominated nucleobase incorporated in double stranded DNA.

Transferring hot-electrons generated in the plasmonic nanoparticles to a different material is a very interesting topic, principally because it can lead to a better understanding of the plasmon-induced interfacial properties. Recently Zhang et al. ${ }^{15}$ showed that hot-electrons can be transferred from Au nanoparticles to semiconductors, and can go as far as $10 \mathrm{~nm}$ in semiconductors or $1 \mathrm{~nm}$ in metals and also perform the dimerization reaction of 4aminothiophenol very efficiently. In this case, the authors showed that the generated hot- 
electrons are very quickly converted to heat in the metal, while in the semiconductor they can have a longer life time enabling a long-range transmission. On the other hand, Kim et al. ${ }^{16}$ showed that hot-electrons can be transferred by multi-step hopping through an insulating layer of aliphatic chains self-assembled on Au nanoparticle surfaces over more than six carbons. Another interesting photocatalyst assembly was reported by Ma et al. ${ }^{17}$ where the authors used DNA to self-assemble $\mathrm{TiO}_{2}$ nanoparticles with $\mathrm{CdS}$ nanorods, enabling electrons to be transferred from the $\mathrm{TiO}_{2}$ to the $\mathrm{CdS}$ and facilitating $\mathrm{CO}_{2}$ reduction. DNA in this case is used as an electron transfer mediator or a molecular scale conductive wire, even helping to promote better charge separation and increase the reaction yield. In this regard, we hypothesize that DNA can serve as an efficient mediator to conduct hot-electrons generated from plasmonic nanoparticles and that these hot-electrons will remain reactive to perform chemical reactions.

DNA is nowadays one of the most versatile biomolecules to obtain 2-D and 3-D nanostructures. Due to the very unique base pair arrangement it is possible to create a wide variety of assemblies. ${ }^{18-21}$ One important technique is DNA-based crystal engineering, where DNA is the responsible to form and maintain the structure while the nanoparticle is the rigid and directional entity, generating the so-called nanoparticle superlattices. Crystal engineering with DNA has already enabled the fabrication of superlattices with a high degree of ordering with many different crystallographic orientations, ${ }^{22}$ both using isotropic and anisotropic nanoparticles, ${ }^{23,24}$ and even crystalline and epitaxial films were already obtained. ${ }^{25-28}$ Due to the large efforts in crystallizing nanoparticles, many of the design and self-assembly rules governing the creation of superlattices are already well-described in the literature. ${ }^{22,29}$ One of the most promising applications of DNA-based nanoparticle superlattices is the possibility of generating plasmonic materials with well-defined functionality. ${ }^{24,25,30,31}$ However in most of these cases DNA has limited functionality and many of its properties regarding what the DNA chains are capable of is still limited. Recently it was shown that the superlattices formed using DNA and $20 \mathrm{~nm}$ gold nanoparticles presented electrochemical conductivity and a very fast charge transfer rate which is mainly dependent on the DNA molecule. ${ }^{32,33}$

Herein we self-assembled $60 \mathrm{~nm}$ silver nanoparticle (AgNP) superlattices to probe hotelectron induced reactions in single brominated nucleotides inserted into the DNA chains far from the nanoparticle surface. The superlattice design allowed us to provide electromagnetic enhancement enough to track the reduction of the brominated nucleotide by SERS in a single point modification scale. Also due to the addressability offered byDNA it was possible to put the modified base at very specific positions, allowing us to check the possibility of transferring 
hot-electrons through DNA, which could enable further hot-electron induced reactions to occur far from the nanoparticle surface. The obtained results showed that DNA is capable of transferring the hot-electrons generated in the AgNPs to the reaction probe molecule far from the nanoparticle surface. It also indicates that the use of DNA-based superlattices provides a good platform to carry out hot-electron driven reactions in a very controlled way. This is also the first time we show that brominated nucleobases inserted into DNA can be decomposed by plasmonically generated hot-electrons far (i.e. $>5.5 \mathrm{~nm}$ ) from the nanoparticle surface.

\section{Results and Discussion}

\section{Design of the nanoparticle-DNA superlattices}

The present study revolves around two central questions: (i) Is it possible to transfer hot-carriers from AgNP through DNA? (ii) Is this hot-carrier able to react with a specific molecule? A 3D- superlattice is an ensemble of nanoparticles which is organized in a way that resembles an atomic crystal arrangement, i.e., there is a certain distance between the nanoparticle centers and there is a repetition unity in three dimensions. In this way, the nanoparticles are not simply aggregated but are arranged in a manner imparting much more stable and uniform electromagnetic field than in a random aggregate. To accomplish this, we used DNA to act as the functional linking unit scaffolding the nanoparticles in place. In doing so, we select the DNA sequences with some considerations in mind:

- The DNA sequences should be short enough to allow for the formation of plasmonic hot-spots, enabling to track the reaction pathway using SERS;

- The hot-carrier participation in the reaction should come from only one nanoparticle, so the reactant molecule should be insulated from the carriers coming from other nanoparticles;

- The DNA should only contain one modified base per sequence, in order to know the correct positioning of the base in relation to the nanoparticle surface.

Taking into consideration these points and also the existing rules to self-assemble the superlattices, we designed a superlattice system composed of 4 different DNA sequences, which are referred to as Thiol A and B and Linker A and B. A model of the current system is illustrated in Figure 1. The two thiol sequences present a dithiolserinol group at the 3 ' end to allow the easy modification of silver nanoparticles ( $\mathrm{SH}$ groups at the end of the sequences), followed by a short flexible sequence composed of 5 cytosine $(\mathrm{dC})$, which also interact with the nanoparticle surface, bringing the first base after it closer to the nanoparticle. After this 
there is a sequence containing 12 bases only composed of cytosine $(\mathrm{dC})$ and guanine $(\mathrm{dG})$, those 12 bases are complementary to 12 bases present at the linker sequences forming double helices. In the linker sequence there is a $\mathrm{C}_{12}$ aliphatic chain after the complementary sequence separating the first 12 bases from the next 8 bases, which stick to the complementary DNA present on the other sequence. The $\mathrm{C}_{12}$ aliphatic chain was chosen since it is long enough to hinder the charge transfer from the nanoparticles while at the same time imparting flexibility to the sequences towards proper assembly. In Figure 1, the 4 different DNA strands and the positions of modifications related to the nanoparticle surfaces are shown. The used sequences are given in Table 1.

The design chosen provides a distance between nanoparticle surfaces of around 10 to $13 \mathrm{~nm}$, which can generate a strong enough plasmonic coupling to allow the detection of the single DNA base modifications by SERS, as can be observed on Figure $2 \mathrm{~A}$ and $\mathrm{B}$. The modified base 8 -bromo-adenosine ( $8 \mathrm{BrdA}$ ) was chosen as the reactive probe molecule, which is shown as a modified purple base in the Figure 1. Previous results from our group showed that the 8-bromo-adenine nucleobase is decomposed by hot-electron transfer from nanoparticles and that the signal from both the reactant and product present a very strong SERS signal, allowing us to track the reaction by Raman spectroscopy. ${ }^{12,13}$ The chemical structures of $8 \mathrm{BrdA}$ and the products adenosine and a bromide anion are given in Figure 1.

Another advantage of $8 \mathrm{BrdA}$ is that it can easily be inserted into the oligomer during the synthesis without hindering the double helix formation later. In this way, $8 \mathrm{BrdA}$ is inserted into specific positions of the DNA sequence Thiol A, and at the same time a complementary $\mathrm{T}$ was inserted into the Linker sequences. 8BrdA was inserted into the positions 1, 3, 7 and 12 of the Thiol A sequences, corresponding to approximately 1.5, 2.5, 3.9 and $5.5 \mathrm{~nm}$ separation between the nanoparticle surface and the reactant molecule. A sample without $8 \mathrm{BrdA}$ is used as control for the test of the stability of DNA. SEM images (Figure 1C) confirm that the nanoparticles are self-assembled in a tightly packed configuration, and that the superlattices are three dimensional assemblies which are larger than $1 \mu \mathrm{m}$ in diameter, in such way that the Raman laser spot is completely probing the reaction in the superlattice and not the $\mathrm{SiO}_{2}$ substrate. 

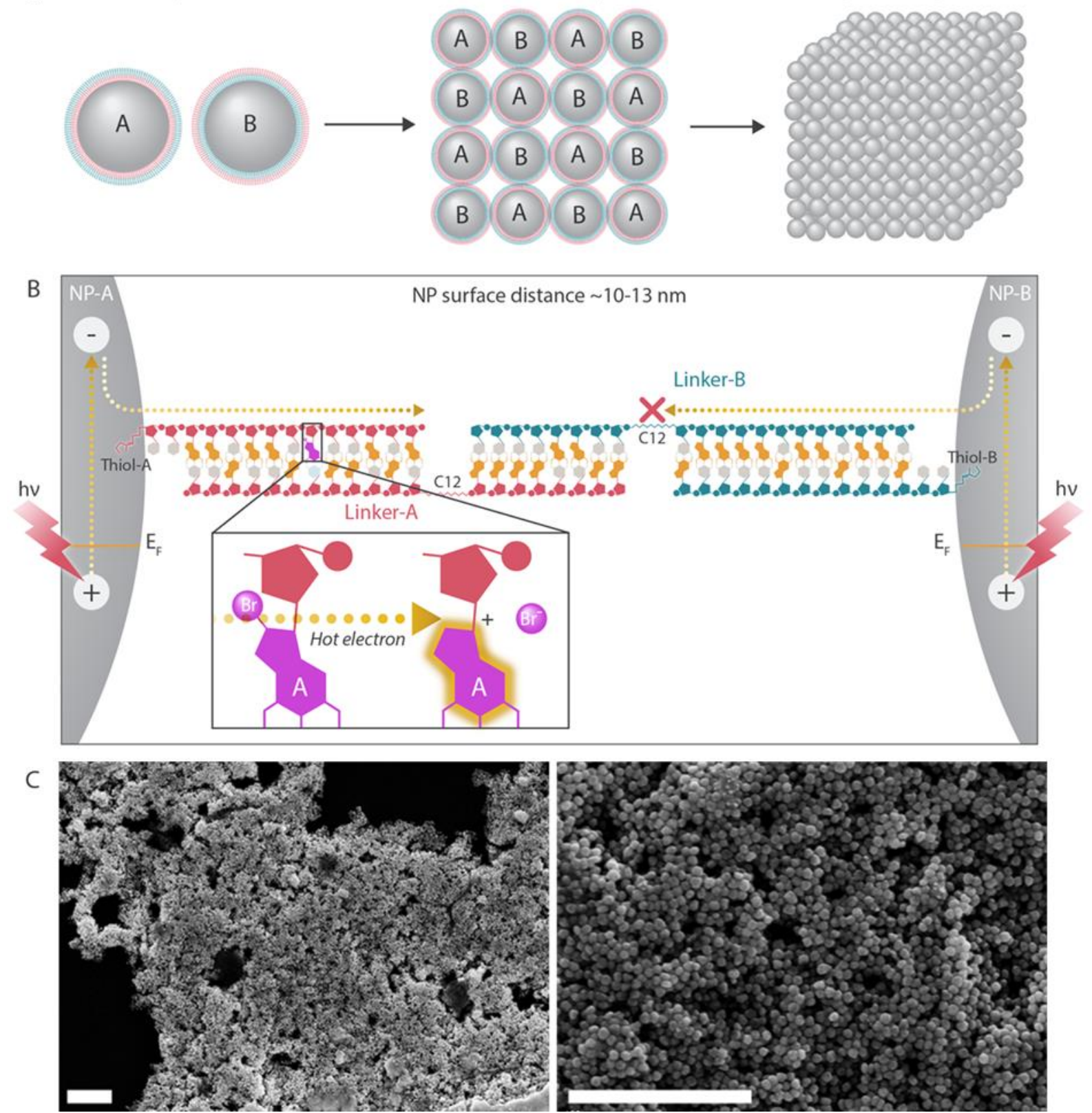

Figure 1. Schematic showing the AgNP superlattice. A) AgNP modified with DNA sequence $\mathrm{A}$ and $\mathrm{B}$, are mixed together and by base-pairing complementarity it can self-assembled in large superlattices. B) The DNA base pairing between the sequences $\mathrm{A}$ and $\mathrm{B}$, bring the two nanoparticles together, with a distance between 10 to $13 \mathrm{~nm}$. The $8 \mathrm{BrdA}$ modification is inserted into sequence $A$, here shown in purple at the $7^{\text {th }}$ position. Upon light irradiation the nanoparticles generate a hot-electron and hot-hole pair. The hot-electron can then be injected into the DNA double helix, enabling the hot-electron reaction. All nanoparticles in the lattice can generate hot-carriers, but due to the $\mathrm{C}_{12}$ chain each nanoparticle is insulated from the other, allowing the precise separation distance control. C) SEM images of the formed AgNP superlattices, the white scale bar represents $1 \mu \mathrm{m}$, which is similar in size to the laser spot size used to probe the reaction. 

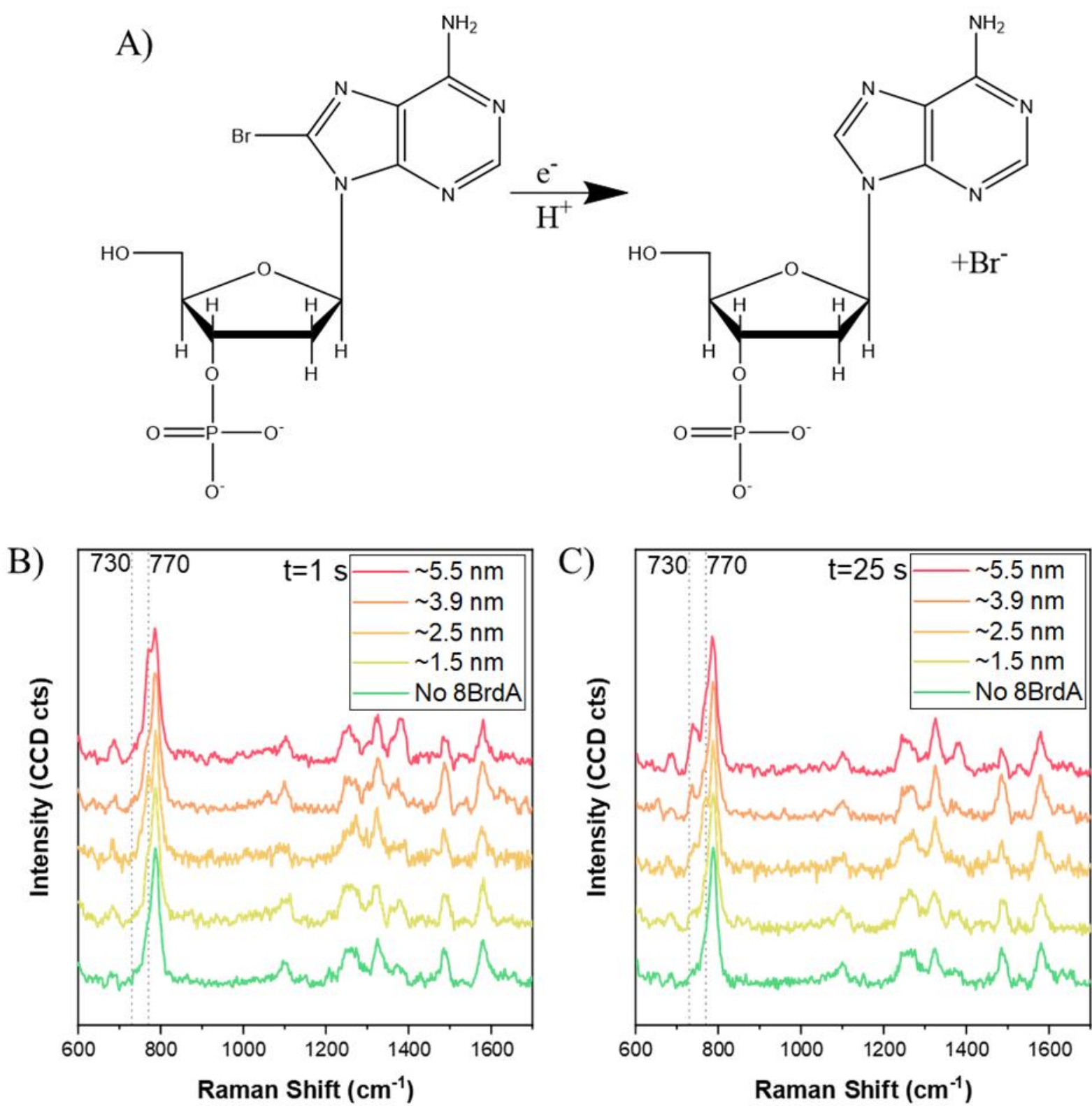

Figure 2. Hydrodehalogenation reaction tracked using SERS. A) Due to the nanoparticle irradiation hot-electrons are ejected which can interact with the 8BrdA cleaving the $\mathrm{C}$-Br bond at C-8 position, generating adenosine base and a bromide anion. B) SERS spectra recorded at $\mathrm{t}=1 \mathrm{~s}$, just after the irradiation is started for five different samples containing $8 \mathrm{BrdA}$ at different positions indicated by distance $(\mathrm{nm})$ from nanoparticle surface as mentioned in the legend The samples containing 8BrdA present a peak or shoulder at the region around $770 \mathrm{~cm}^{-1}$. B) SERS spectrum collected after $25 \mathrm{~s}$ of irradiation. In the samples containing $8 \mathrm{BrdA}$ the presence of a peak around $730 \mathrm{~cm}^{-1}$ corresponding to the adenosine ring-breathing vibration is clearly observed with no clear signature of the same in the control sample (green). The laser intensity for these spectra is $200 \mu \mathrm{W}$.

The choice of $8 \mathrm{BrdA}$ provides the possibility to detect single modifications by SERS based on its characteristic ring-breathing mode even when $8 \mathrm{BrdA}$ is surrounded by $\mathrm{CG}$ bases. Figure $1 \mathrm{~A}$ shows the presence of the ring breathing mode of $8 \mathrm{BrdA}$ in the region around 770 
$\mathrm{cm}^{-1}$ in the SERS spectra, while the region around $730 \mathrm{~cm}^{-1}$ is clear of vibrational bands. After $25 \mathrm{~s}$ of irradiation (Figure 2B) the spectra shows the presence of a very well-defined vibration at $730 \mathrm{~cm}^{-1}$ corresponding to the ring-breathing mode of adenosine, while the peak at $770 \mathrm{~cm}^{-}$ ${ }^{1}$ is diminished allowing us to track the decomposition of the $8 \mathrm{BrdA}$ and the appearance of adenosine over time. Additionally, we observe less intense vibrations related to the DNA backbone principally in the region between 1000 and $1600 \mathrm{~cm}^{-1}$ as seen in Figure $2 \mathrm{~A}$ and B. ${ }^{34}$ It is important to stress that the peaks corresponding to DNA backbone remain unaffected during the reaction and the reaction takes place specifically at the $8 \mathrm{BrdA}$. That is to say, the rest of the DNA is not under the impact of the hot-electrons for the laser power used.

\section{Decomposition of $8 B r d A$ in DNA}

Since we could observe that after a certain time the $8 \mathrm{BrdA}$ is decomposed and that the adenosine is being formed, we then tracked the reaction for a longer period collecting SERS spectra throughout the reaction in order to study or comment on the reaction rate of hydrodehalogenation reaction under different experimental parameter. Figure $3 \mathrm{~A}$ shows timedependent spectra between 0 and $70 \mathrm{~s}$ of irradiation, and it is possible to observe the instantaneous appearance of the adenosine peak at $734 \mathrm{~cm}^{-1}$ with decrease in peak intensity of $8 \mathrm{BrdA}$ at $765 \mathrm{~cm}^{-1}$ with time. . We tracked the reaction over a period of $800 \mathrm{~s}$, and the intensity map is shown in Figure 3B. The map in Figure 3B shows an intense vibration of 8BrdA in the beginning of the reaction that rapidly decreases with continuous increase of the adenosine peak over time. During the reaction we also observe the presence of flare emissions which are not associated with the reaction, but that were recently attributed to atomic dislocations of the metal atoms in the nanoparticles. ${ }^{35}$

It is possible to obtain the kinetics from the time traces for both the decomposition of $8 \mathrm{BrdA}$ and the generation of adenosine from all the collected spectra. The time trace data extracted from Figure 3B is shown in Figure 3C. The plot in Figure $3 \mathrm{C}$ shows that the $8 \mathrm{BrdA}$ vibration intensity decreases very rapidly, and that the first $100 \mathrm{~s}$ of irradiation are sufficient to decrease the counts by around 300, after which the drop in peak intensity becomes slow until the irradiation is stopped. On the other hand, a concomitant rise in peak at $765 \mathrm{~cm}^{-1}$ corresponding to adenosine vibration can be observed with time. Recently Liu et al. ${ }^{36}$ proposed a very interesting mechanism for the plasmon-mediated hydrodehalogenation reaction of 8bromo-adenine and the many reaction steps. 

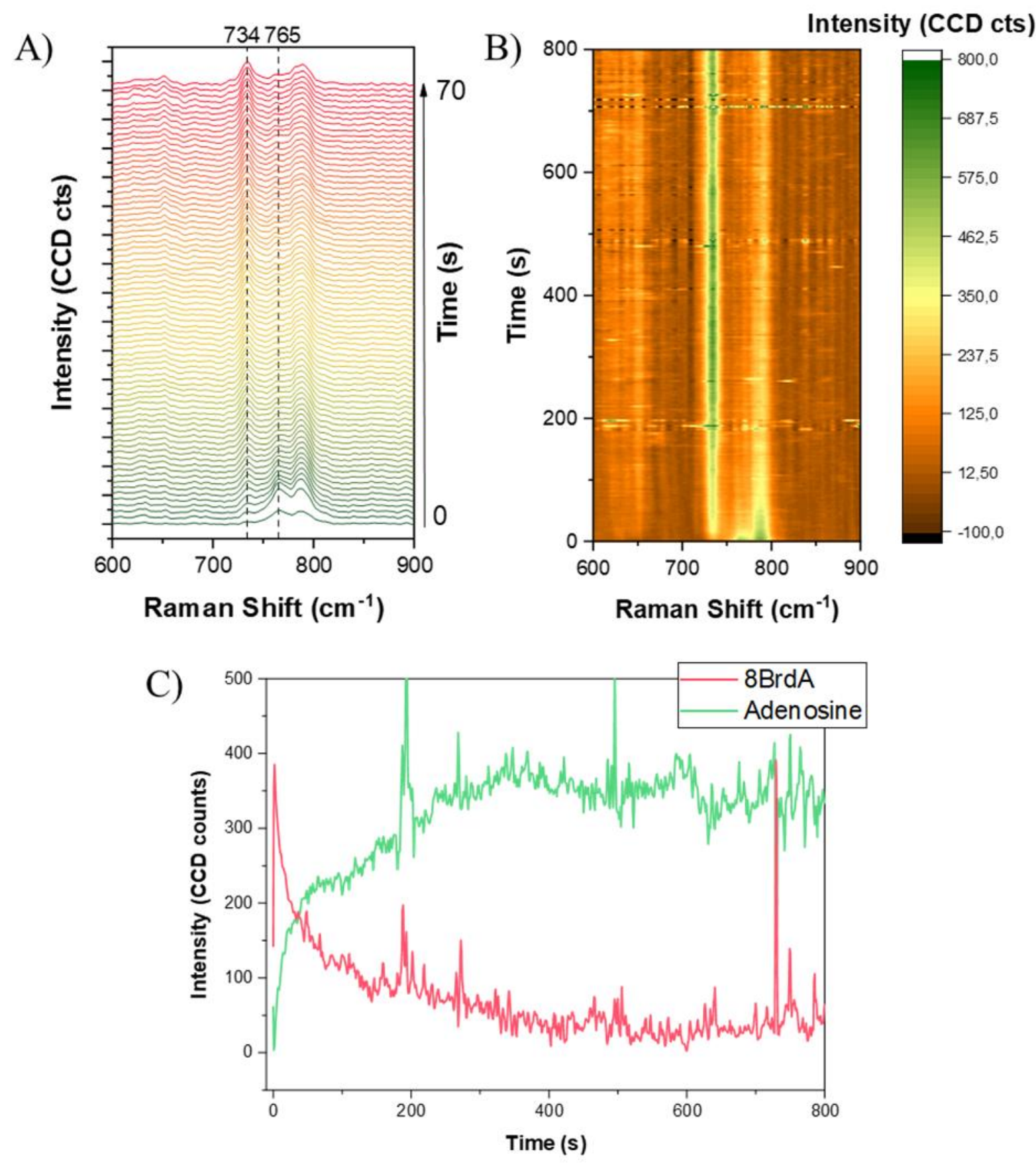

Figure 3. 8BrdA decomposition over time. A) Time series SERS spectra of the sample with $8 \mathrm{BrdA}$ at $\sim 2.5 \mathrm{~nm}$ in the ring-breathing region (the two marked bands are related to the $8 \mathrm{BrdA}$ at $765 \mathrm{~cm}^{-1}$ and to Adenosine at $734 \mathrm{~cm}^{-1}$ ) over the period of $70 \mathrm{~s}, 1 \mathrm{~s}$ of integration time. B) Time series map for the sample with $8 \mathrm{BrdA}$ at $\sim 2.5 \mathrm{~nm}$ showing the progress of the reaction during $800 \mathrm{~s}$. C) Reaction time traces extracted from the time series shown in graph B, reflecting the kinetics of $8 \mathrm{BrdA}$ decay at $765 \mathrm{~cm}^{-1}$ and the formation of adenosine due to increase peak intensity at $734 \mathrm{~cm}^{-1}$.

One of the most interesting properties of DNA is the possibility to transfer charges along the double helix. Such property was first observed by Jacqueline Barton in the 90s by a 
series of photophysical studies of DNA intercalating molecules and base pair damage, and later it was also extensively studied by electrochemical methods. ${ }^{37-41}$ But even with many different contributions still nowadays it is a topic under intense debate principally regarding the flexibility of $\mathrm{DNA}^{42}$ and also the possibility of the charge transfer to happen in the sugar backbone and not in the base pairs. ${ }^{43}$ At the same time, it was also reported that DNA could be used to transfer hot-electrons generated by Ag or AuNP, and that the excess charges could quench a fluorophore or lead to DNA degradation, but in those cases the nanoparticles were irradiated with high intensity pulsed lasers. ${ }^{44,45}$ Here in our case, we used a continuous wave laser to track the hot-electron driven reaction showing that DNA is able to act as a conductive wire for hot-electron transfer and that the hot-electrons are able to selectively break bonds in modified DNA bases.

To further confirm and prove the charge transfer process, we modified the DNA sequence by incorporating the $8 \mathrm{BrdA}$ (purple base) in the stitch region in between two $\mathrm{C}_{12}$ chains (Scheme 1). As explained before, an aliphatic $\mathrm{C}_{12}$ chain should be long enough to fully hinder the conduction of electrons from the nanoparticles to the probing zone. The time series spectra and map of the samples corresponding to insulated 8BrdA is shown in Figure 4A and 4B. Interestingly, it is possible to observe an intense $8 \mathrm{BrdA}$ peak at $765 \mathrm{~cm}^{-1}$, which is still fully visible after $100 \mathrm{~s}$ of irradiation, while the peak for the adenosine appears only after $50 \mathrm{~s}$ of irradiation, but with very low peak intensity. This is in contrast to when compared with the data shown in Figure 3A where the adenosine peak appears right after the irradiation showing a strong intensity peak.

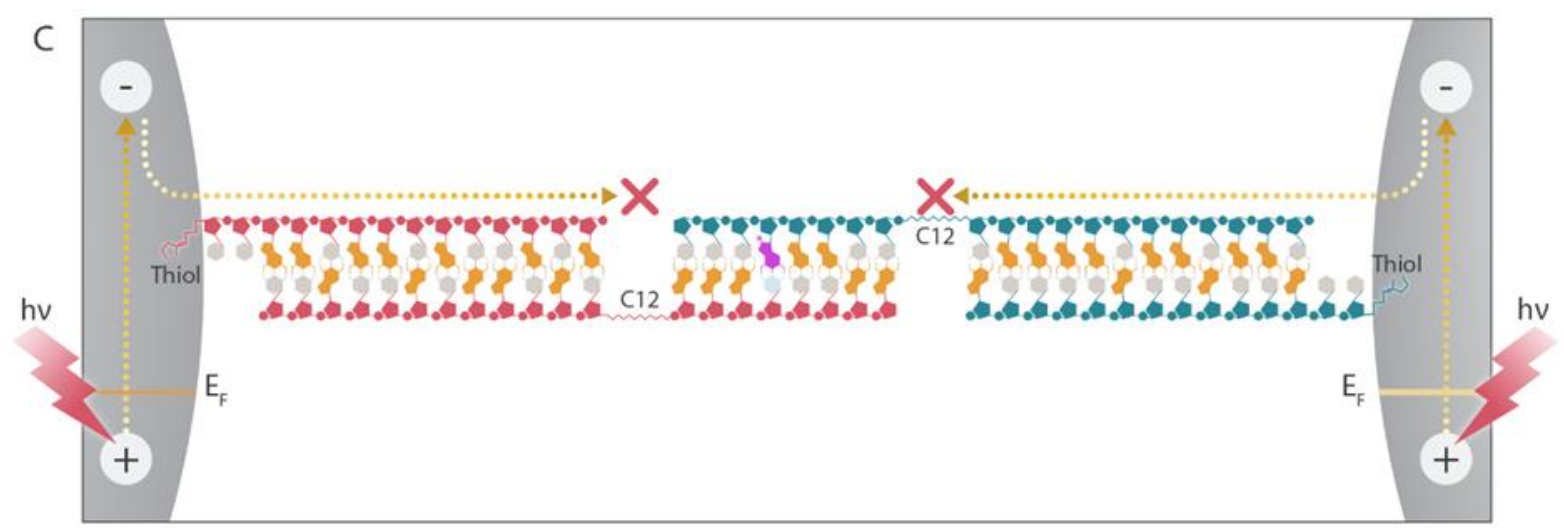

Scheme 1. Schematic of the control experiment. In this case 8BrdA (shown in purple) is included between the two $\mathrm{C}_{12}$ chains which hinder the hot-electron passage from the nanoparticles, preventing the decomposition of the probe molecule. 
From the map shown in Figure 4B, the kinetic time traces can be extracted for both 8BrdA decomposition and adenosine formation, which are then compared to the case where the $8 \mathrm{BrdA}$ is connected to the DNA sequence exposing it to the hot-electron source. In this case we compare with the sample A12 where the $8 \mathrm{BrdA}$ is the farthest away from the nanoparticle surface. This comparison is given in Figure 4C and 4D. The data comparison shows that the $\mathrm{C}_{12}$ chains do hinder the hot-electron passage and that the $8 \mathrm{BrdA}$ reaction does not proceed. The slight decrease in $8 \mathrm{BrdA}$ intensity over long time and also the increase of the adenosine vibration is probably not due to a DEA reaction, but possibly the plasmonically generated heat that can also induce reactions which can lead to molecular decomposition. The distinction between the hot-electron and the heat driven reactions is usually very difficult since both processes are happening at the same time, and both can also lead to the same reaction pathway. Herein we show that the use of a DNA wire can help to disentangle both contributions.

So far, we showed that DNA can be used to self-assemble nanoparticles into superlattices which are useful for SERS analysis, and that the hot-electron is being transferred through DNA far away from the nanoparticle surface and that it can be used to drive reactions within DNA. In the next section, the kinetics of the hot-electron driven reaction will be discussed, focusing on the decomposition process of 8BrdA, and the relationship between the experimentally determined kinetic constant to its position in the DNA sequence and the contribution of the laser power to the reaction. 

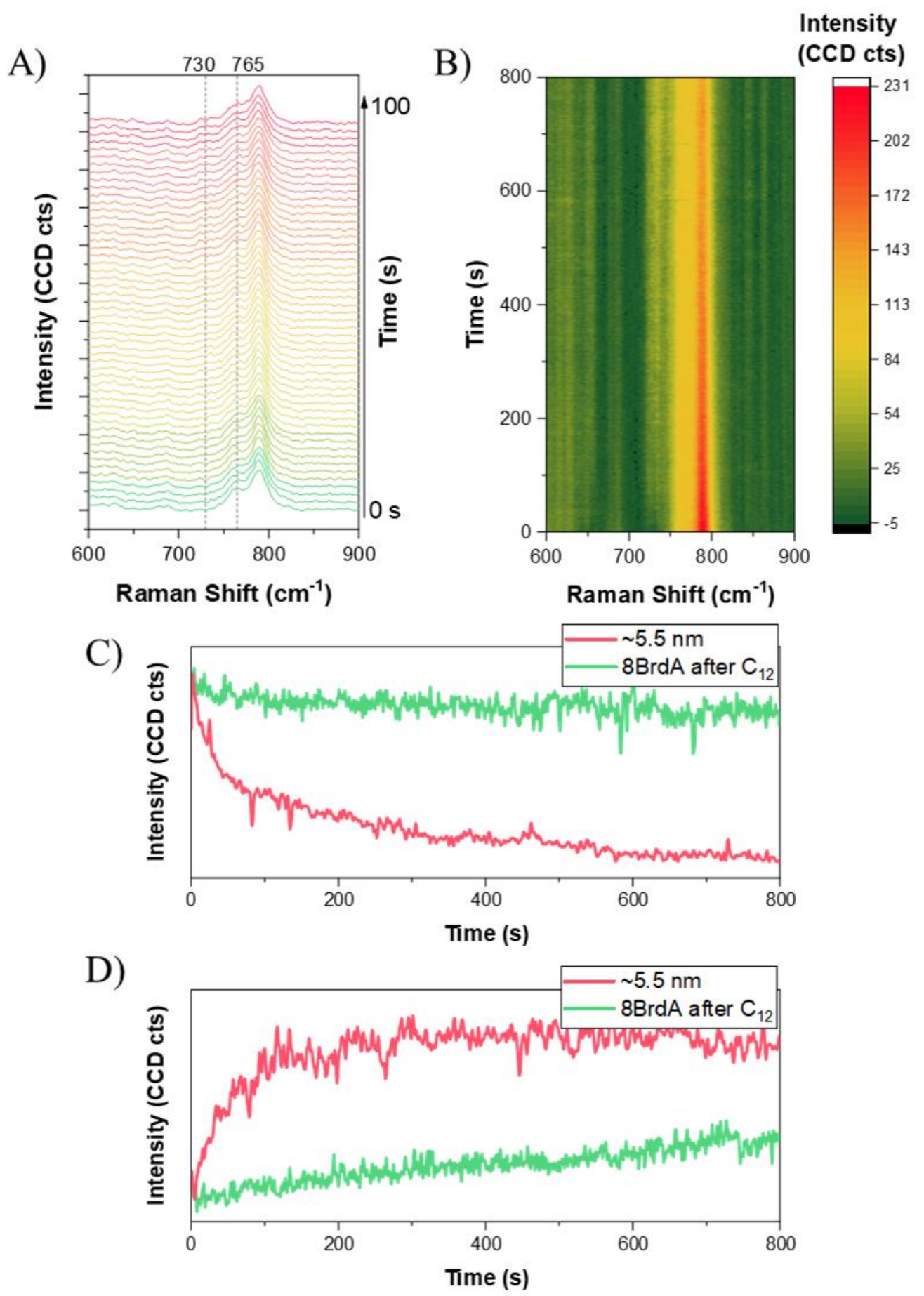

Figure 4. Control experiment of the insulated 8BrdA. A) Timed spectra in the ring-breathing vibration mode of the sample with $8 \mathrm{BrdA}$ located between the $\mathrm{C}_{12}$ chain. The two marked bands are related to the $8 \mathrm{BrdA}$ at $765 \mathrm{~cm}^{-1}$ and to Adenosine at $730 \mathrm{~cm}^{-1}$ over the period of 100 $\mathrm{s}, 1 \mathrm{~s}$ of integration time. B) Time series SERS map of the sample with the $8 \mathrm{BrdA}$ after the $\mathrm{C}_{12}$ chain, showing the progress of the $8 \mathrm{BrdA}$ decomposition reaction upon laser irradiation. $\mathrm{C}$ and D) show the comparison of the experimental reaction time traces for the region of $8 \mathrm{BrdA}(\mathrm{C})$ and Adenosine (D) vibrations. The control experiment where $8 \mathrm{BrdA}$ is insulated is compared to the time traces for the sample with $8 \mathrm{BrdA}$ at $\sim 5.5 \mathrm{~nm}$ (the farthest possible position of $8 \mathrm{BrdA}$ regarding the nanoparticle surface). The conditions for the spectra acquisition are $1 \mathrm{~s}$ integration time and $500 \mu \mathrm{W}$ of laser power in the focal plane. 


\section{Probe Molecule Position and Laser power dependence}

To assess the reaction under different experimental reaction condition and parameters, we differ the probe molecular position with respect to surface and monitor their reaction kinetics under different laser power. As discussed before, due to the many possible steps which could involve the formation of adenosine we will focus our discussion on the decomposition of $8 \mathrm{BrdA}$. We have modelled the reaction of 8-bromoadenine with hot-electrons before by a dissociative electron attachment (DEA) mechanism. ${ }^{14}$ In this mechanism, an electron is captured at a specific energy to form a transient negative ion (TNI), which can dissociate very fast in the time scale of $10 \mathrm{fs}$, resulting in the adenine nucleobase, the structure of the molecules we are probing here were shown in Figure 2A. The fitting procedure used to obtain the kinetics constants is given in the supporting information. Herein we used a second order fractal kinetics equation to fit the obtained kinetics time traces.

The consideration about the fractal system arises from the nanoparticle organization in the nanoscale and the intrinsic inhomogeneity of the SPR excitation in the system. On a nonfractal regime, the reaction rate constant $k$ would be the same all around the nanoparticle surface. However, the close proximity between the nanoparticles creates the so-called hotspots, which are regions that can promote a faster reaction rate, but since this is not homogeneously spread over the nanoparticle surface but constrained in some regions, it can create regions with different $k$ that vary over time. After the initial consumption of the probe molecules located at the most intense hot-spots $\left(k_{1}\right)$, the rest of the molecules on the nanoparticle surface will react but slower compared to the hot-spot reaction $\left(k_{2}, k_{3}, k_{4}, \ldots, k_{n}\right)$, creating a gradient of rate constants in the system, and the relative contribution of each $k$ is dependent on the time such as shown by Schürmann and Bald. ${ }^{12}$ The reaction kinetics in our case follows a second order fractal kinetics rate law:

$$
[8 B r d A]=\frac{[8 B r d A]_{0} *(1-h)}{(1-h)+[8 B r d A]_{0} * k_{2 F} * t^{1-h}},(\text { Equation } 1)
$$

where $[8 \mathrm{BrdA}]$ is the intensity of the $8 \mathrm{BrdA}$ peak in the Raman spectra, [ $8 \mathrm{BrdA}]_{0}$ is the initial intensity of the $8 \mathrm{BrdA}$ peak in the Raman spectra, $k_{2 F}$ is the second order fractal rate constant, $t$ is the time and $h$ is the fractal dimension term. This equation can fit the data directly extracted from the Raman spectra, and from the fit the second order fractal rate constant is obtained, the derivation of this equation is given in the SI. 
The first point of our discussion here is the position of the probe in the DNA chain. The data showing the extracted time traces for the samples with different $8 \mathrm{BrdA}$ positions are given in Figure 5A. For the control where no 8BrdA is present in the DNA chain, we obtain a straight line without any decrease in intensity (at the $\sim 770 \mathrm{~cm}^{-1}$ region of the spectra), indicating that DNA is stable under the experimental conditions employed. Upon the inclusion of the 8BrdA in the DNA double helix it is now possible to observe and track the decomposition of the probe molecule. The data in Figure 5A shows that the decomposition of 8BrdA takes place for all the cases when $8 \mathrm{BrdA}$ is included in the DNA.

From the kinetic analysis we obtain the value of the second order fractal kinetics rate constant $k_{2 F}$. In Figure $5 \mathrm{~B}$ it is shown how the $k_{2} \mathrm{~F}$ varies depending on the probe position on the DNA chain and hence with the distance to the nanoparticle surface. This is shown for different laser powers, which will be discussed later. The distance dependence of $k_{2}$ follows a U-shaped profile, i.e., the rate constant is high when the probe is the closest to the surface (about $1.5 \mathrm{~nm}$ ), then decreases in the middle position $(2.5$ and $3.9 \mathrm{~nm})$ and is again very high when furthest away from the surface (around $5.5 \mathrm{~nm}$ ). Like discussed before the conduction through DNA is still a topic under debate, but we can do some considerations regarding the topic here.

One very important aspect of our system is the DNA sequence used. The sequences used here are very rich in CG base pairs which have a very interesting conduction aspect. One of the most accepted mechanisms of DNA conduction assumes that the conduction occurs by a mixed process involving both tunneling and multistep hopping. ${ }^{46-48}$ Giese et al ${ }^{48,49}$ observed that hopping through the CG base pairs is very much favored due to the lower redox potential of the $\mathrm{G}$ nucleobase. The possibility of the AT pair to conduct holes by hopping was later tested by the same group. More recently Xiang et al. ${ }^{50}$ showed that having an ordered array of CG base pairs can enhance DNA conductivity a bit due to the HOMOs of the stacked Gs being delocalized over several $\mathrm{G}$ bases, like in our case where some $\mathrm{G}$ bases are stacked helping the electron transfer. The most interesting property of the hopping-like mechanism is that it is distance independent, i.e., the charge carrier can be transferred through long DNA sequences without losing all its energy. ${ }^{41}$ And in our results we can observe that even with the probe $\sim 5.5$ $\mathrm{nm}$ far from the nanoparticle surface the reaction is still happening at a similar rate as when it is the closest at $\sim 1.5 \mathrm{~nm}$. Another aspect of DNA charge transfer was studied recently by Kékedy-Nagy and Ferapontova, ${ }^{51}$ who showed a very interesting asymmetry of the DNA mediated charge transfer towards the reduction of methylene blue, and found that charge 
transfer in the forward direction is faster than in the backward direction, an indication that DNA can also be used to avoid charge recombination, increasing the reaction rate for the generation of the products since the electrons can live longer within the DNA double helix.
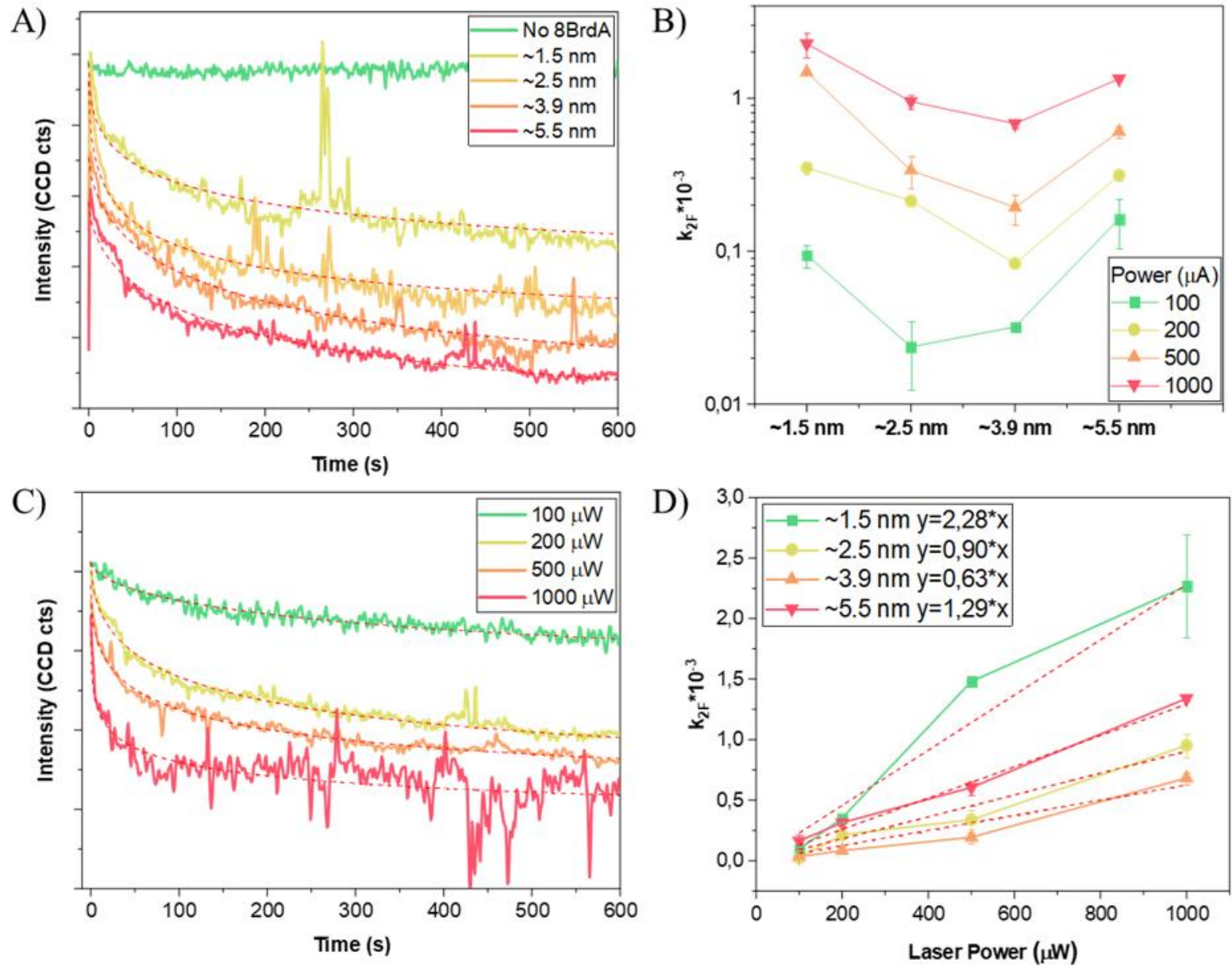

Figure 5. Effect of the base placement and laser power on the $8 \mathrm{BrdA}$ decomposition reaction. A) Comparison of the experimental reaction traces for different samples containing 8BrdA. The data are offset for better visualization, the data were extracted from the experiment obtained using $200 \mu \mathrm{W}$ of laser power. The red traced curves show the fit of the data using the second order fractal kinetics (equation 1). B) Comparison between the $k_{2} \mathrm{~F}$ obtained from the fits in A) and the position of 8BrdA in the DNA for different laser powers. C) Comparison of the experimental kinetic traces using different laser powers. The red traced curves show the fit of the data using the second order fractal kinetics (Equation 1). The data shown here is related to the sample containing 8BrdA at $\sim 5.5 \mathrm{~nm}$ from the AgNP surface. D) Experimental relationship between the $k_{2 \mathrm{~F}}$ with the laser power for the 4 samples containing the $8 \mathrm{BrdA}$ at different positions. The red traced lines show the linear fit of the points for each one of the samples, and the linear equation describing each dataset is given in the figure legend.

The U-shape profile of the observed $k_{2 \mathrm{~F}}$ can be explained as follows: Recently Li and Han demonstrated by simulations that the stacking of the DNA bases can alter the long range charge transfer through DNA. ${ }^{52}$ The authors suggest that due to the stacking of the orbitals, it is possible to form a potential well with depth of $0.1 \sim 0.3 \mathrm{eV}$ in vacuo, which stabilizes the base 
pairs in the middle of the sequence while at the same time can lead to a lower stabilization of the base pairs in the edge of the sequence, which in turn could enhance the reactivity of such bases towards an external agent, such as the nanoparticle generated hot-electrons. ${ }^{53}$ The present data suggests that $8 \mathrm{BrdA}$ located at the edges is subject to a lower stabilization due to the incomplete stacking by the $\pi$-orbital clouds of the DNA double helix, making it more prone to the reaction with hot-electrons. At the same time the probe molecules located in the middle of the sequence are much more stabilized by the other bases around it, making it harder to be decomposed by the hot-electrons thereby decreasing the reaction rate. The data presented in Figure 5B also shows the dependence of the reaction rate on the laser power used for the irradiation, which will be discussed next.

The laser power affects a plasmon-induced reaction in two different ways: on the one hand a higher laser power results in generation of more hot-electrons increasing the reaction rate by increasing the number of reactants hot-electrons, on the other hand it can be converted to heat due to the thermalization of the hot-carriers and the reaction is faster due to the thermal contribution. ${ }^{1,4,54}$ Disentangling both contributions is very hard principally because both processes happen at the same time (at least in the time scales discussed here in this work). The time traces for the sample containing 8BrdA far from the AgNP surface (i.e., $~ 5.5 \mathrm{~nm}$ ) at different laser powers is given in Figure $5 \mathrm{C}$. The decomposition rate of $8 \mathrm{BrdA}$ is directly proportional to the increase in laser power, i.e., at higher laser powers the reaction proceeds faster compared to low laser powers. At the same time, we observe that at high laser powers $(1000 \mu \mathrm{W})$ the reaction reaches a plateau in less than $100 \mathrm{~s}$, and that the continued irradiation leads to different processes observed by the very intense noise signal after $300 \mathrm{~s}$. All the curves at different laser power were fitted using the second order fractal integrated rate law, and the obtained $k_{2 \mathrm{~F}}$ values at different laser power is given in Figure 5D. The data on Figure 5B and $\mathrm{D}$ are the same but the different representations give us hints on different processes.

The data in Figure 5D suggests that the reaction rate increases linearly with the laser power, especially for the samples with $8 \mathrm{BrdA}$ at $\sim 2.5, \sim 3.9$ and $\sim 5.5 \mathrm{~nm}$. The average value for the slope of the linear fit for these samples is 0.94 , an indication that increasing the laser power directly increases the reaction rate by the same order of increase, while for the sample with $8 \mathrm{BrdA}$ very close to the $\mathrm{AgNp}$ surface $(\sim 1.5 \mathrm{~nm})$ the slope of the linear fit is 2.28 . This is an indication that the reaction with slope closer to 1 represents a hot-electron triggered reaction, while a higher slope value indicates that plasmonically generated heat is also contributing. Baffou and Quidant ${ }^{55,56}$ showed that the heat dissipation for spherical nanoparticles under CW 
irradiation follows a $1 / r$ distribution, i.e., the temperature decreases with increasing distance from the NP surface and the influence of thermal effects gets less as we can observe in Figure 5B and 5D. In conclusion, the high-rate constant observed for the sample with 8BrdA closer to the NP surface is not only due to a limited nucleotide stabilization at the beginning of the sequence, but also due to some contribution of heat.

We can observe the lowest rate for the sample with $8 \mathrm{BrdA}$ at $\sim 2.5 \mathrm{~nm}$ at a laser power of $100 \mu \mathrm{W}$, but increasing the laser power to $200 \mu \mathrm{W}$ increases its rate more than that of the probe at $3.9 \mathrm{~nm}$, making this sample now the slowest and keeping this pattern throughout the experiment. This shift is possibly due to the heat generated by the particle spreading further from the particle surface; however, it does not increase as fast as when the probe molecule is at $\sim 1.5 \mathrm{~nm}$ due to the possible stabilization effect that the DNA base pairing introduces to the system. At $\sim 3.9 \mathrm{~nm}$ the reaction is not very much influenced by the heat generated by the particles, the reaction is slower than the other positions, an indication that DNA provides a stabilized structure, and that the hot-electrons are probably distributed through the chain which decreases the reactivity of the $8 \mathrm{BrdA}$. Finally, when $8 \mathrm{BrdA}$ is very far from the nanoparticle surface it is only influenced by the hot-electron transfer, and since it is at the end of the chain it does not have the full stabilization of DNA, making its reaction rate higher compared to the cases when the probe is in the middle of the chain, but lower than the closest one since it is not subject to the extra energy input coming from the heated surface.

\section{Conclusions}

To conclude, herein we showcase the use of self-assembled nanoparticle superlattices for plasmon-induced reactions, and for the first time we demonstrate that DNA can be used to transfer hot-electrons far from the nanoparticle surface. DNA allows to build nanoparticle lattices with a very short interparticle spacing, and it also provides a suitable scaffold for controlled placement of modifications. We have used 8BrdA known for its simple decomposition reaction pathway compared to other molecules, requiring only one electron and one proton for the reaction. The hydrodehalogenation reaction of $8 \mathrm{BrdA}$ at different positions followed a second order fractal kinetics rate law with $k_{2 \mathrm{~F}}$ dependent on the position of the probe molecule in the DNA sequence and also on the laser power employed for irradiation. More than that, we show that by the correct placement of the probe molecule it is possible to control the hot-electron driven pathway over the thermal pathway, principally due to hot-electron transfer through DNA. 
The results shown here opens up many possibilities for plasmon-induced chemical reactions. So far, the reactions were confined to the surface of the plasmonic nanoparticle, while we show that this could occur further away from the nanoparticle surface using DNA as charge-conducting wire. Further understanding of the DNA-nanoparticle interaction at the interface and charge injection into DNA needs to be gained in future experiments. The possibility of transferring electrons from the DNA to molecules which are interacting with but are not covalently bound to DNA also needs to be explored in further studies. Optimizing the charge extraction process to carry out reactions that require more than one hot-carrier also needs further attention. In the future, we hope that the study presented here serves as a basis to better understand plasmon-induced reactions which do not require direct contact with the nanoparticle surface.

\section{Authors contribution}

SKJr and IB conceived the study, SKJr prepared all the samples, characterized and measured the decomposition reaction, SKJr, AD and IB analyzed and discussed the data. All authors contributed to the manuscript writing.

\section{Acknowledgements}

SKJr acknowledges the São Paulo Research Foundation (FAPESP fellowships 2016/14507-8 and 2018/17831-6), Brazil, for financial support. This research was also supported by the European Research Council (ERC; consolidator grant no. 772752), the University of Potsdam, and by the German Federal Ministry of Education and Research (grant no. 03Z22A512).

\section{Experimental Section}

\section{Materials}

All chemicals were of highest purity available and were used without further purification. Sodium citrate, TAE buffer 10X, sodium dodecylsulfate (SDS), $\mathrm{HCl}, \mathrm{NaOH}$, $\mathrm{NaCl}$ were obtained from Sigma-Aldrich. Silver nanoparticles with diameter of $60 \mathrm{~nm}$ stabilized by sodium citrate were obtained from Nanocomposix. The DNA sequences were all obtained from Metabion $\mathrm{GmbH}$, and all the sequences were purified by HPLC and confirmed with mass spectrometry. Water was purified by a Milli-Q system.

Table 1. DNA sequences used in this work. 


\begin{tabular}{|c|c|}
\hline Name & Sequence $\left(5^{\prime} \rightarrow 3^{\prime}\right)$ \\
\hline Thiol A & GCC CCG CCG CCG CCCCC-(SH) \\
\hline Thiol B & GCG CCG CGG CGG-CCCCC-(SH) \\
\hline Thiol A1 & GCC CCG CCG CC(8BrdA) CCCCC-(SH) \\
\hline Thiol A3 & GCC CCG CCG (8BrdA)CG CCCCC-(SH) \\
\hline Thiol A7 & GCC CC(8BrdA) CCG CCG CCCCC-(SH) \\
\hline Thiol A12 & (8BrdA)CC CCG CCG CCG CCCCC-(SH) \\
\hline Linker A & CGG CGG CGG GGC-(C $\left.\mathrm{C}_{12}\right)-\mathrm{CCG}$ GCC CC \\
\hline Linker B & CCG CCG CGG CGC-(C 12$)-$ GG GGC CGG \\
\hline Linker A1 & TGG CGG CGG GGC-(C 12$)-C C G$ GCC CC \\
\hline Linker A3 & CGT CGG CGG GGC-(C 12$)$-CCG GCC CC \\
\hline Linker A7 & CGG CGG TGG GGC-( $\left.\mathrm{C}_{12}\right)$-CCG GCC CC \\
\hline Linker A12 & CGG CGG CGG GGT-(C 12$)-C C G$ GCC CC \\
\hline Linker AM & CGG CGG CGG GGC-( $\left.\mathrm{C}_{12}\right)-\mathrm{CCG}$ G(8BrdA)C CC \\
\hline Linker BM & CCG CCG CGG CGC-(C 12$)-G G$ GTC CGG \\
\hline
\end{tabular}

\section{Nanoparticle Modification and Superlattice Self-Assembly}

To modify the AgNP with the thiolated DNA sequences, we adapted the low $\mathrm{pH}$ method protocol. In this procedure, the dispersion of nanoparticles and DNA are submitted to a lower $\mathrm{pH}$ by the addition of citric acid at $\mathrm{pH} 3 .{ }^{57}$ For $1 \mathrm{~mL}$ of $60 \mathrm{~nm}$ AgNP, 2\% SDS was added to a concentration of $0.02 \%$ and shaken for $20 \mathrm{~min}$ at $37{ }^{\circ} \mathrm{C}$. Then $8000 \mathrm{X}$ excess thiolated DNA was added i.e, around $4 \mu \mathrm{L}$ of a $100 \mu \mathrm{M}$ DNA-SH solution, and was left shaking for 20 min to fully interact with the AgNP. After $20 \mathrm{~min}, \mathrm{pH}$ of the solution was changed to 3 by the addition of $500 \mathrm{mmol} \mathrm{L}^{-1}$ of citric acid, i.e, around 20 to $40 \mu \mathrm{L}$ is sufficient for the change. After more 10 min shaking at $37^{\circ} \mathrm{C}$, the $\mathrm{pH}$ of the solution is restored to around 7 by the addition of the same volume of 10X TAE buffer. Using these conditions the concentration of $\mathrm{Na}^{+}$in solution is around 100 to $200 \mathrm{mmol} \mathrm{L}^{-1}$. This step adds the thiolated DNA to the nanoparticle surface in a very high yield.

Next to the modified nanoparticle solution, 16000X excess of the linker sequence is added in solution, and the mixture is heated to $75^{\circ} \mathrm{C}$ for $15 \mathrm{~min}$ while shaking. This temperature promotes the full melting of the DNA oligomers. After this the AgNP solution is cooled off by $5^{\circ} \mathrm{C}$ every 10 min untill a temperature of $30^{\circ} \mathrm{C}$ is reached which is then left for shaking at this temperature overnight. Next the nanoparticles are washed by centrifugation 5 times to remove any aggregate and non-bound DNA present in solution. After every wash the supernatant is removed and the volume is made up with $50 \mathrm{mmol} \mathrm{L}^{-1} \mathrm{NaCl}$ with $0.02 \%$ SDS. After every 
wash the solution is sonicated in a bath sonicator for 1 min to break any nanoparticle aggregates. At the end the nanoparticle concentration is quantified by UV-Vis spectrometry.

From the freshly modified nanoparticles the superlattices are assembled by mixing the nanoparticles modified with the sequence $\mathrm{A}$ and the ones modified with the sequence $\mathrm{B}$ in equimolar amounts. To the solution more $\mathrm{NaCl}$ is added to reach the concentration between 100 to $150 \mathrm{mmol} \mathrm{L}^{-1}$ of $\mathrm{Na}^{+}$. The mixtures are then inserted into a thermo cycler, that is heated up to $50{ }^{\circ} \mathrm{C}$ for $15 \mathrm{~min}$, and is later cooled down to $20^{\circ} \mathrm{C}$ over the course of 24 hours. If the superlattice self-assembly proceeds correctly the formation of precipitates is observed, on the other hand the solution will keep its yellow color if the superlattice is not formed. Or, Successful formation of superlattice self-assembly is ensured by precipitation of the dispersion, on the other hand unsuccessful self-assembly leaves behind a yellow color nanoparticle dispersion.

\section{Raman Spectra acquisition and data analysis}

SERS spectra have been recorded using a confocal Raman microscope (WITec 300 $\alpha$ ) equipped with an upright optical microscope. For Raman excitation, laser light at $\lambda=633 \mathrm{~nm}$ was used that was coupled into a single-mode optical fiber and focused through a 50× objective (Olympus MPlanFL N, NA = 0.75) to a spot size of about $1000 \mathrm{~nm}$. The laser power was varied between $100,200,500$ and $1000 \mu \mathrm{W}$ at the focal plane, and the integration time was $1 \mathrm{~s}$. The kinetics were followed between 5 to 15 minutes for each sample, i.e. each curve is based on 300 to 900 different spectra. Each sample was measured at least 3 times, and the results shown here are the average of all the different Raman measurements, i.e., all the spectra collected were averaged over time, and the time traces are extracted directly from the average data. 


\section{Bibliographic References}

(1) Jain, P. K. Taking the Heat off of Plasmonic Chemistry. J. Phys. Chem. C. 2019, 123 (40), 24347-24351.

(2) Koopman, W.; Sarhan, R. M.; Stete, F.; Schmitt, C. N. Z.; Bargheer, M. Decoding the Kinetic Limitations of Plasmon Catalysis: The Case of 4-Nitrothiophenol Dimerization. Nanoscale 2020, 12 (48), 24411-24418.

(3) Mukherjee, S.; Libisch, F.; Large, N.; Neumann, O.; Brown, L. V.; Cheng, J.; Lassiter, J. B.; Carter, E. A.; Nordlander, P.; Halas, N. J. Hot Electrons Do the Impossible: Plasmon-Induced Dissociation of H 2 on Au. Nano Lett. 2013, 13 (1), 240-247.

(4) Miliutina, E.; Guselnikova, O.; Soldatova, N. S.; Bainova, P.; Elashnikov, R.; Fitl, P.; Kurten, T.; Yusubov, M. S.; Švorčík, V.; Valiev, R. R.; Chehimi, M. M.; Lyutakov, O.; Postnikov, P. S. Can Plasmon Change Reaction Path? Decomposition of Unsymmetrical Iodonium Salts as an Organic Probe. J. Phys. Chem. Lett. 2020, 11 (14), 5770-5776.

(5) Novello, P.; Varanasi, C. V.; Liu, J. Effects of Light on Catalytic Activities and Lifetime of Plasmonic Au Catalysts in the CO Oxidation Reaction. ACS Catal. 2019, 9 (1), 578-586.

(6) Liu, L.; Wang, Y.; Fu, W. Highly Selective Detection of Sulfide through Poisoning Silver Nanoparticle Catalysts. Sensors Actuators, B Chem. 2017, 247, 414-420.

(7) Szczerbiński, J.; Gyr, L.; Kaeslin, J.; Zenobi, R. Plasmon-Driven Photocatalysis Leads to Products Known from E-Beam and X-Ray-Induced Surface Chemistry. Nano Lett. 2018, 18 (11), 6740-6749.

(8) Heck, C.; Kanehira, Y.; Kneipp, J.; Bald, I. Amorphous Carbon Generation as a Photocatalytic Reaction on DNA-Assembled Gold and Silver Nanostructures. Molecules 2019, 24 (12), 2324.

(9) Gellé, A.; Jin, T.; De La Garza, L.; Price, G. D.; Besteiro, L. V.; Moores, A. Applications of Plasmon-Enhanced Nanocatalysis to Organic Transformations. Chem. Rev. 2020, 986-1041.

(10) Choi, H. K.; Park, W. H.; Park, C. G.; Shin, H. H.; Lee, K. S.; Kim, Z. H. MetalCatalyzed Chemical Reaction of Single Molecules Directly Probed by Vibrational Spectroscopy. J. Am. Chem. Soc. 2016, 138 (13), 4673-4684.

(11) Schürmann, R.; Ebel, K.; Nicolas, C.; Milosavljević, A. R.; Bald, I. Role of Valence Band States and Plasmonic Enhancement in Electron-Transfer-Induced Transformation of Nitrothiophenol. J. Phys. Chem. Lett. 2019, 10 (11), 3153-3158.

(12) Schürmann, R.; Bald, I. Real-Time Monitoring of Plasmon Induced Dissociative Electron Transfer to the Potential DNA Radiosensitizer 8-Bromoadenine. Nanoscale 2017, 9 (5), 1951-1955.

(13) Dutta, A.; Schürmann, R.; Bald, I. Plasmon Mediated Decomposition of Brominated Nucleobases on Silver Nanoparticles - A Surface Enhanced Raman Scattering (SERS) Study. Eur. Phys. J. D 2020, 74 (1), 19.

(14) Schürmann, R.; Tanzer, K.; Dạbkowska, I.; Denifl, S.; Bald, I. Stability of the Parent 
Anion of the Potential Radiosensitizer 8-Bromoadenine Formed by Low-Energy $(<3$ EV) Electron Attachment. J. Phys. Chem. B 2017, 121 (23), 5730-5734.

(15) Zhang, H.; Wei, J.; Zhang, X. G.; Zhang, Y. J.; Radjenovica, P. M.; Wu, D. Y.; Pan, F.; Tian, Z. Q.; Li, J. F. Plasmon-Induced Interfacial Hot-Electron Transfer Directly Probed by Raman Spectroscopy. Chem 2020, 6 (3), 689-702.

(16) Kim, Y.; Wilson, A. J.; Jain, P. K. The Nature of Plasmonically Assisted Hot-Electron Transfer in a Donor-Bridge-Acceptor Complex. ACS Catal. 2017, 7 (7), 4360-4365.

(17) Ma, K.; Yehezkeli, O.; He, L.; Cha, J. N. DNA for Assembly and Charge Transport Photocatalytic Reduction of CO 2 . Adv. Sustain. Syst. 2018, 2 (4), 1700156.

(18) Laramy, C. R.; O’Brien, M. N.; Mirkin, C. A. Crystal Engineering with DNA. Nat. Rev. Mater. 2019, 201-224.

(19) Tapio, K.; Bald, I. Multifunctional Materials The Potential of DNA Origami to Build Multifunctional Materials The Potential of DNA Origami to Build Multifunctional Materials. Multifunct. Mater. 2020, 3 (3), 032001.

(20) Kogikoski, S.; Paschoalino, W. J.; Kubota, L. T. Supramolecular DNA Origami Nanostructures for Use in Bioanalytical Applications. TrAC - Trends in Analytical Chemistry. 2018, 88-97.

(21) Seeman, N. C.; Sleiman, H. F. DNA Nanotechnology. Nat. Rev. Mater. 2017, 3, 17068.

(22) Macfarlane, R. J.; Lee, B.; Jones, M. R.; Harris, N.; Schatz, G. C.; Mirkin, C. A. Nanoparticle Superlattice Engineering with DNA. Science 2011, 334 (6053), 204-208.

(23) O’Brien, M. N.; Jones, M. R.; Lee, B.; Mirkin, C. A. Anisotropic Nanoparticle Complementarity in DNA-Mediated Co-Crystallization. Nat. Mater. 2015, 14 (8), 833-839.

(24) Sun, L.; Lin, H.; Park, D. J.; Bourgeois, M. R.; Ross, M. B.; Ku, J. C.; Schatz, G. C.; Mirkin, C. A. Polarization-Dependent Optical Response in Anisotropic NanoparticleDNA Superlattices. Nano Lett. 2017, 17 (4), 2313-2318.

(25) Ross, M. B.; Ku, J. C.; Blaber, M. G.; Mirkin, C. A.; Schatz, G. C. Defect Tolerance and the Effect of Structural Inhomogeneity in Plasmonic DNA-Nanoparticle Superlattices. Proc. Natl. Acad. Sci. U. S. A. 2015, 112 (33), 10292-10297.

(26) Ross, M. B.; Bourgeois, M. R.; Mirkin, C. A.; Schatz, G. C. Magneto-Optical Response of Cobalt Interacting with Plasmonic Nanoparticle Superlattices. J. Phys. Chem. Lett. 2016, 7 (22), 4732-4738.

(27) Gabrys, P. A.; Seo, S. E.; Wang, M. X.; Oh, E.; Macfarlane, R. J.; Mirkin, C. A. Lattice Mismatch in Crystalline Nanoparticle Thin Films. Nano Lett. 2018, 18 (1), $579-585$.

(28) Senesi, A. J.; Eichelsdoerfer, D. J.; Macfarlane, R. J.; Jones, M. R.; Auyeung, E.; Lee, B.; Mirkin, C. A. Stepwise Evolution of DNA-Programmable Nanoparticle Superlattices. Angew. Chemie - Int. Ed. 2013, 52 (26), 6624-6628.

(29) Macfarlane, R. J.; O’Brien, M. N.; Petrosko, S. H.; Mirkin, C. A. Nucleic AcidModified Nanostructures as Programmable Atom Equivalents: Forging a New "Table 
of Elements." Angew. Chem. - Int. Ed. 2013, 5688-5698.

(30) Ross, M. B.; Ku, J. C.; Lee, B.; Mirkin, C. A.; Schatz, G. C. Plasmonic Metallurgy Enabled by DNA. Adv. Mater. 2016, 28 (14), 2790-2794.

(31) Young, K. L.; Ross, M. B.; Blaber, M. G.; Rycenga, M.; Jones, M. R.; Zhang, C.; Senesi, A. J.; Lee, B.; Schatz, G. C.; Mirkin, C. A. Using DNA to Design Plasmonic Metamaterials with Tunable Optical Properties. Adv. Mater. 2014, 26 (4), 653-659.

(32) Kogikoski, S.; Kubota, L. T. Electrochemical Behavior of Self-Assembled DNA-Gold Nanoparticle Lattice Films. Electrochem. commun. 2018, 90, 51-55.

(33) Kogikoski, S.; Kubota, L. T. Electron Transfer in Superlattice Films Based on SelfAssembled DNA-Gold Nanoparticle. Electrochim. Acta 2019, 318, 931-936.

(34) Guerrini, L.; Krpetić, Ž.; Van Lierop, D.; Alvarez-Puebla, R. A.; Graham, D. Direct Surface-Enhanced Raman Scattering Analysis of DNA Duplexes. Angew. Chem. - Int. Ed. 2015, 54 (4), 1144-1148.

(35) Carnegie, C.; Urbieta, M.; Chikkaraddy, R.; de Nijs, B.; Griffiths, J.; Deacon, W. M.; Kamp, M.; Zabala, N.; Aizpurua, J.; Baumberg, J. J. Flickering Nanometre-Scale Disorder in a Crystal Lattice Tracked by Plasmonic Flare Light Emission. Nat. Commun. 2020, 11, 682.

(36) Liu, J.; Cai, Z. Y.; Sun, W. X.; Wang, J. Z.; Shen, X. R.; Zhan, C.; Devasenathipathy, R.; Zhou, J. Z.; Wu, D. Y.; Mao, B. W.; Tian, Z. Q. Plasmonic Hot Electron-Mediated Hydrodehalogenation Kinetics on Nanostructured Ag Electrodes. J. Am. Chem. Soc. 2020, 142 (41), 17489-17498.

(37) Murphy, C. J.; Arkin, M. R.; Jenkins, Y.; Ghatlia, N. D.; Bossmann, S. H.; Turro, N. J.; Barton, J. K. Long-Range Photoinduced Electron Transfer through a DNA Helix. Science 1993, 262 (5136), 1025-1029.

(38) Arkin, M. R.; Stemp, E. D. A.; Holmlin, R. E.; Barton, J. K.; Hörmann, A.; Olson, E. J. C.; Barbara, P. F. Rates of DNA-Mediated Electron Transfer between Metallointercalators. Science 1996, 273 (5274), 475-480.

(39) Kelley, S. O.; Barton, J. K.; Jackson, N. M.; Hill, M. G. Electrochemistry of Methylene Blue Bound to a DNA-Modified Electrode. Bioconjug. Chem. 1997, 8 (1), 31-37.

(40) Drummond, T. G.; Hill, M. G.; Barton, J. K. Electrochemical DNA Sensors. Nat. Biotechnol. 2003, 1192-1199.

(41) Slinker, J. D.; Muren, N. B.; Renfrew, S. E.; Barton, J. K. DNA Charge Transport over 34 Nm. Nat. Chem. 2011, 3 (3), 228-233.

(42) Dauphin-Ducharme, P.; Arroyo-Currás, N.; Plaxco, K. W. High-Precision Electrochemical Measurements of the Guanine-, Mismatch-, and Length-Dependence of Electron Transfer from Electrode-Bound DNA Are Consistent with a ContactMediated Mechanism. J. Am. Chem. Soc. 2019, 141 (3), 1304-1311.

(43) Zhuravel, R.; Huang, H.; Polycarpou, G.; Polydorides, S.; Motamarri, P.; Katrivas, L.; Rotem, D.; Sperling, J.; Zotti, L. A.; Kotlyar, A. B.; Cuevas, J. C.; Gavini, V.; Skourtis, S. S.; Porath, D. Backbone Charge Transport in Double-Stranded DNA. Nat. Nanotechnol. 2020, 15 (10), 836-840. 
(44) Wirth, J.; Garwe, F.; Meyer, R.; Csáki, A.; Stranik, O.; Fritzsche, W. Plasmonically Enhanced Electron Escape from Gold Nanoparticles and Their Polarization-Dependent Excitation Transfer along DNA Nanowires. Nano Lett. 2014, 14 (7), 3809-3816.

(45) Toppari, J. J.; Wirth, J.; Garwe, F.; Stranik, O.; Csaki, A.; Bergmann, J.; Paa, W.; Fritzsche, W. Plasmonic Coupling and Long-Range Transfer of an Excitation along a DNA Nanowire. ACS Nano 2013, 7 (2), 1291-1298.

(46) Genereux, J. C.; Barton, J. K. Mechanisms for DNA Charge Transport. Chem. Rev. 2010, 110 (3), 1642-1662.

(47) Beratan, D. N. Why Are DNA and Protein Electron Transfer So Different? Annu. Rev. Phys. Chem. 2019, 70 (1), 71-97.

(48) Giese, B. Long-Distance Charge Transport in DNA: The Hopping Mechanism. Acc. Chem. Res. 2000, 33 (9), 631-636.

(49) Giese, B.; Amaudrut, J.; Köhler, A. K.; Spormann, M.; Wessely, S. Direct Observation of Hole Transfer through DNA by Hopping between Adenine Bases and by Tunnelling. Nature 2001, 412 (6844), 318-320.

(50) Xiang, L.; Palma, J. L.; Bruot, C.; Mujica, V.; Ratner, M. A.; Tao, N. Intermediate Tunnelling-Hopping Regime in DNA Charge Transport. Nat. Chem. 2015, 7 (3), 221226.

(51) Kékedy-Nagy, L.; Ferapontova, E. E. Directional Preference of DNA-Mediated Electron Transfer in Gold-Tethered DNA Duplexes: Is DNA a Molecular Rectifier? Angew. Chemie - Int. Ed. 2019, 58 (10), 3048-3052.

(52) Li, Z.; Han, K. Long-Range Stacking Effects of Nucleobases in Charge Transfer. ChemRxiv, 2020.

(53) Saito, I.; Takayama, M.; Sugiyama, H.; Nakatani, K.; Tsuchida, A.; Yamamoto, M. Photoinduced DNA Cleavage via Electron Transfer: Demonstration That Guanine Residues Located 5' to Guanine Are the Most Electron-Donating Sites. J. Am. Chem. Soc. 1995, 117 (23), 6406-6407.

(54) Kim, Y.; Dumett Torres, D.; Jain, P. K. Activation Energies of Plasmonic Catalysts. Nano Lett. 2016, 16 (5), 3399-3407.

(55) Baffou, G.; Quidant, R.; García De Abajo, F. J. Nanoscale Control of Optical Heating in Complex Plasmonic Systems. ACS Nano 2010, 4, 709-716.

(56) Baffou, G.; Quidant, R. Thermo-Plasmonics: Using Metallic Nanostructures as NanoSources of Heat. Laser Photonics Rev., 2013, 171-187.

(57) Liu, B.; Liu, J. Methods for Preparing DNA-Functionalized Gold Nanoparticles, a Key Reagent of Bioanalytical Chemistry. Anal. Meth. 2017, 2633-2643. 